\title{
Transcription factor UvMsn2 is important for vegetative growth, conidiogenesis, stress response, mitochondrial morphology and pathogenicity in the rice false smut fungus Ustilaginoidea virens
}

\author{
Yuandi $\mathrm{Xu}^{\dagger}$, Shuang $\mathrm{Wu}^{\dagger}$, Zhaomeng Yu, Emily Kolojane Moeketsi, Zhixiang Yang, Zhengguang Zhang and \\ Haifeng Zhang ${ }^{*}$ (D)
}

\begin{abstract}
Transcription factors (TFs) play critical roles in the control of development and pathogenicity of phytopathogens by directly or indirectly regulating the expression of downstream genes. Here, we identified and characterized a zinc finger TF UvMsn2 in Ustilaginoidea virens, a homolog of MoMsn2 from the rice blast fungus. Heterogenous complementation assay revealed that UvMsn2 fully restored the defects of the Momsn2 mutant in vegetative growth, conidiation and pathogenicity. Deletion of UvMsn2 in U. virens led to a reduction of the pathogen in vegetative growth, aerial hyphae and conidiation. Additionally, the $\Delta U v m s n 2$ mutant displayed defects in conidial morphology and germination, as well as mitochondrial morphology. Pathogenicity and toxicity assays revealed that the $\Delta U v$ vmsn2 mutant was non-pathogenic and less inhibitory to rice seed germination. The $\Delta U v m s n 2$ mutant showed different sensitivity to various stresses. Further microscopic examination found that UvMsn2 was localized in both cytosol and nucleus, and translocated from cytosol to nucleus under the treatment of $\mathrm{NaCl}$. Our results demonstrate that UvMsn2 is a critical TF that regulates the vegetative growth, conidiogenesis, stress response, mitochondrial morphology and virulence in the rice false smut fungus.
\end{abstract}

Keywords: Transcription factor UvMsn2, Conidiogenesis, Stress response, Mitochondrial morphology, Pathogenicity, Ustilaginoidea virens

\section{Background}

Eukaryotes have evolved complex and precise mechanisms for perception and transduction of extracellular signals to adapt to environmental stresses, including transcriptional regulation of genes (Rajvanshi et al. 2017). Transcriptional regulation by a diverse array of transcription factors (TFs) is a major mechanism for

\footnotetext{
* Correspondence: hfzhang@njau.edu.cn

tYuandi Xu and Shuang Wu contributed equally to this work. Department of Plant Pathology, College of Plant Protection, Nanjing Agricultural University, and Key Laboratory of Integrated Management of Crop Diseases and Pests, Ministry of Education, Nanjing 210095, China
}

modulating cellular development and differentiation in response to physiological or environmental stimuli (Zhang et al. 2014). Different types of TFs participate in various cellular activities and signal responses through controlling transcriptional activities of downstream genes (Zhang et al. 2014). In budding yeast Saccharomyces cerevisiae, two zinc finger proteins, Msn2 and Msn4, are key TFs that respond to multiple stresses by regulating the transcription of stress-response genes (Estruch and Carlson 1993). Both proteins bind specifically to the stress response element AGGGG or GGGGA when exposed to stress environment (Stewart-Ornstein et al.

(c) The Author(s). 2021 Open Access This article is licensed under a Creative Commons Attribution 4.0 International License, which permits use, sharing, adaptation, distribution and reproduction in any medium or format, as long as you give

appropriate credit to the original author(s) and the source, provide a link to the Creative Commons licence, and indicate if changes were made. The images or other third party material in this article are included in the article's Creative Commons licence, unless indicated otherwise in a credit line to the material. If material is not included in the article's Creative Commons licence and your intended use is not permitted by statutory regulation or exceeds the permitted use, you will need to obtain permission directly from the copyright holder. To view a copy of this licence, visit http://creativecommons.org/licenses/by/4.0/ 
2013). Over $90 \%$ genes were positively regulated by Msn2/4 under heat, osmotic and carbon starvation conditions (Gasch et al. 2000); and the regulation of these genes is dependent on the translocation of Msn2/4 from the cytosol to the nucleus (Rajvanshi et al. 2017).

Under glucose-rich conditions or in cells at the log phase of growth, Msn2 is distributed in the cytosol, but is translocated from the cytosol to the nucleus under stress or glucose depletion conditions (Gorner et al. 2002). Msn2 also shows a carbon source-dependent localization pattern and plays a role in fatty acid oxidation in yeast (Rajvanshi et al. 2017). Non-favorable carbon sources including oleate, galactose and glycerol can induce the translocation of Msn2 from the cytosol to the nucleus. Once shifted to nucleus, Msn2 targets several $\beta$-oxidation genes that are involved in fatty acid oxidation to regulate fatty acid metabolism (Rajvanshi et al. 2017). Additionally, Msn2/4 also plays a role in targeting the rapamycin (TOR) pathway by which to regulate multiple cellular processes in response to nutrients (Crespo and Hall 2002). Inhibition of the TOR pathway leads to increased stress response due to nuclear accumulation of Msn2/4 in yeast (Crespo and Hall 2002). In the rice blast fungus Magnaporthe oryzae, MoMsn2 is distributed in both cytosol and nucleus, and translocated from cytosol to nucleus under salt stress. MoMsn2 controls vegetative growth, conidiogenesis, stress response, cell wall integrity and pathogenicity by regulating the expression of a series of downstream genes (Zhang et al. 2014). Our latest findings indicate that Msn2 targets MoAUH1, a putative 3-methylglutaconyl-CoA hydratase-encoding gene, to govern infectious growth via mitochondrial fusion/fission balance in M. oryzae (Xiao et al. 2021). Meanwhile, the data implicate that MoMsn2 might be involved in maintaining normal function related to mitochondria and peroxisomes in $M$. oryzae, such as glycometabolism, amino acid metabolism or fatty acid metabolism (Xiao et al. 2021). In two entomopathogenic fungi, Msn2 is required for multi-stress responses, conidiation and virulence (Liu et al. 2013).

Rice false smut, caused by the ascomycete fungus Ustilaginoidea virens (Cook) Takahashi (Teleomorph: Villosiclava virens), is an emerging serious disease that occurs in the majority of rice-growing areas worldwide (Sun et al. 2020). Since the availability of the genomic sequence of $U$. virens and establishment of CRISPR/ Cas9-based genome editing system, a growing number of molecular genetic studies on this fungal pathogen have been performed (Liang et al. 2018; Guo et al. 2019). A number of genes that play important roles in the development and virulence of $U$. virens have been identified and well characterized (Sun et al. 2020), including several TFs such as UvHox2, UvPro1 and UvCom1 (Lv et al. 2016; Zheng et al. 2017; Chen et al. 2020b).
However, the zinc finger TF Msn2 has not been identified and characterized in $U$. virens. In this study, we identified UvMsn2 in $U$. virens as a homolog of MoMsn2. Our results demonstrate that UvMsn2 plays important roles in vegetative growth, conidiogenesis, stress response, mitochondrial morphology and pathogenicity in $U$. virens.

\section{Results \\ UvMsn2 is a homolog of MoMsn2 from the rice blast fungus $M$. oryzae}

Our previous study revealed that MoMsn2, a transcription factor from $M$. oryzae, plays pleotropic roles in hyphal growth, conidiogenesis, stress response, mitochondrial morphology and pathogenicity of $M$. oryzae by modulating the expression of a series of genes directly or indirectly (Zhang et al. 2014). Here, we identified a homolog of MoMsn2 in the rice false smut fungus $U$. virens by using a BLAST_P search, and named it UvMsn2. UvMsn2 contains 535 amino acids (aa), and shows $40 \%$ aa identity to MoMsn2. Domain prediction revealed that UvMsn2 possesses two nuclear localization signal sequences (NLS1: 422-456 aa and NLS2: 525-535 aa; http://nls-mapper.iab. keio.ac.jp/cgi-bin/NLS_Mapper_form.cgi, cut-off score: 7.0), and two zinc finger domains ( $\mathrm{C} 2 \mathrm{H} 2: 426-437$ aa and 445-465 aa; https://myhits.sib.swiss/cgi-bin/motif_scan) at its carboxyl terminus, similar to that found in MoMsn2 (Fig. 1a). We then generated the pYF11-UvMSN2 (driven by its native promoter) construct and transformed it into the $\Delta$ Momsn 2 mutant. The resulting heterologous complemented transformants $\triangle M o m s n 2 / L v M S N 2$ were screened by GFP signal and used for phenotypic analysis. It was demonstrated that $U v M S N 2$ almost fully restored the defects of the $\Delta M o m s n 2$ mutant in vegetative growth, conidiation and pathogenicity, and $\triangle M o m s n 2 / U v M S N 2$ showed a very similar colony morphology, sporulation capacity and pathogenicity as that of the wild-type Guy11 of $M$. oryzae (Fig. 1b, c). Meanwhile, the mitochondrial morphology of $\triangle M o m s n 2 / U v M S N 2$ was also restored to that of the wild-type strain (Additional file 1: Figure S1). These results suggest that both the structure and biological role of Msn2 are well conserved in M. oryzae and U. virens.

\section{Targeted-deletion of UVMSN2 and complementation in $U$. virens}

To investigate the biological role of UvMsn2 in the rice false smut fungus, we generated the gene replacement construct pMD19-T-UvMSN2KO containing a hygromycin cassette $(H P H)$ (Additional file 1: Figure S2a) and CRISPR/Cas9 construct pCas9-tRNA-UvMSN2 according to our previous study (Guo et al. 2019). The resulting constructs were analyzed by sequencing and then co-transformed into the protoplasts of the wild-type 


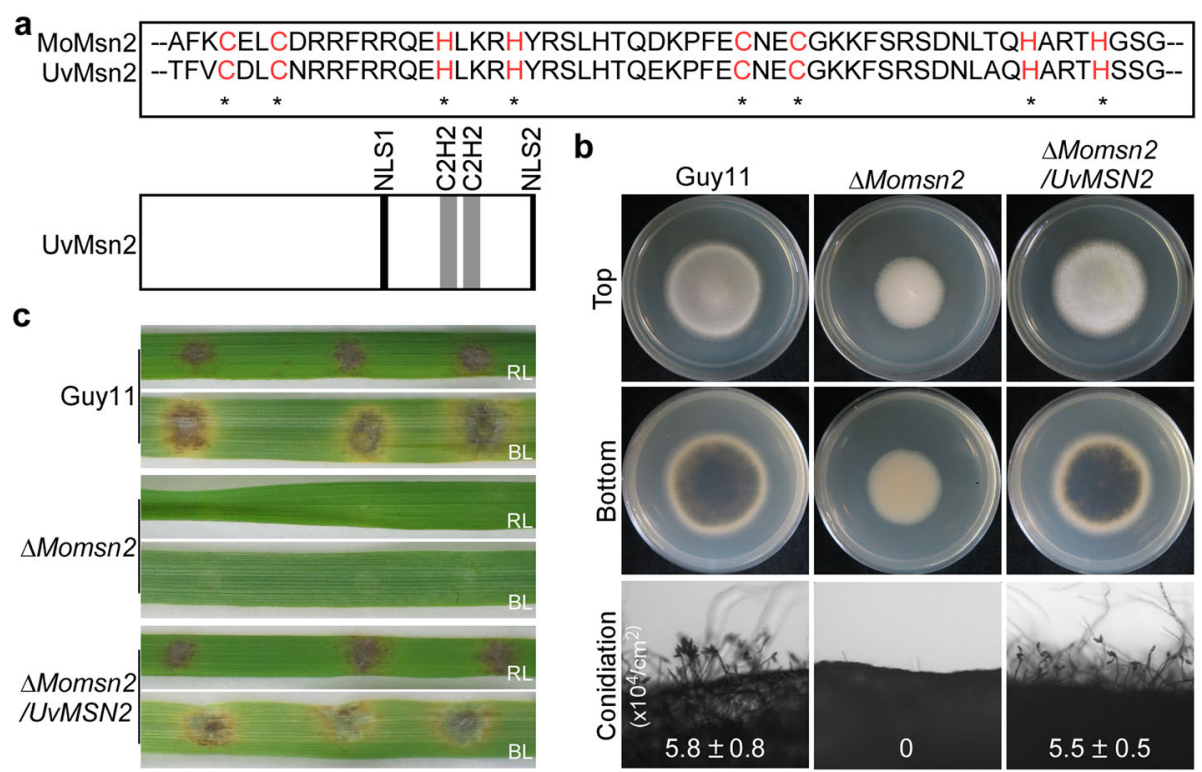

Fig. 1 UvMsn2 is a homolog of MoMsn2 from Magnaporthe oryzae. a Sequence alignment and domain prediction of UvMsn2. b Vegetative growth and conidiation of the M. oryzae wild-type Guy11, Momsn2 mutant and heterologous complemented transformant $\triangle$ Momsn2/UvMSN2. The mean values $\pm S D$ were calculated from three independent experiments. $\mathbf{c}$ Pathogenicity assays on detached rice and barley leaves. The diseased leaves were photographed at 5 and 7 days post-inoculation (dpi) for barley and rice, respectively. RL: rice leaf; BL: barley leaf

HWD-2. A total of 20 hygromycin B-resistant transformants were obtained, of which three candidates were subsequently screened out by PCR. Finally, two candidates were confirmed as UvMSN2 deletion mutants $(\Delta U \nu m s n 2)$ by southern blot analysis, with each having a single copy of HPH cassette (Additional file 1: Figure S2b). For complementation, we generated the pYF11-UvMSN2-GFP construct (containing the entire UvMSN2 gene including its $1.5-\mathrm{kb}$ promoter region) and transformed it into the $\Delta U v m s n 2$ mutant. The resulting bleomycin-resistant transformants with green fluorescent protein (GFP) signals rescued the defects of the $\Delta U v m s n 2$ mutant, and were selected as the final complemented strains, and named $\Delta U v m s n 2 /$ UvMSN2.

\section{UvMsn2 is important for vegetative growth and \\ conidiogenesis in $U$. virens}

We first investigated the role of UvMsn2 in vegetative growth of $U$. virens by inoculating the wild-type HWD2, $\Delta U v m s n 2$ mutant and complemented strain $\triangle U v m s n 2 / U v M S N 2$ onto YT and PDA media. Compared with HWD-2 and $\Delta U v m s n 2 / U v M S N 2$, the $\Delta U v m s n 2$ mutant showed a much smaller colony size with less pigment and aerial hyphae on both YT and PDA media after incubated at $25^{\circ} \mathrm{C}$ for 14 days (Fig. 2a). The colony diameter of the $\Delta U v m s n 2$ mutant was reduced by 51.9 and $46.2 \%$ on YT and PDA, respectively (Fig. 2b). We then assessed the sporulation capacity of the $\Delta U v m s n 2$ mutant, and found that the conidial number was decreased by $76.5 \%$ compared with the wild type (Fig. $2 \mathrm{~d}$ ). Meanwhile, we found that $79.5 \%$ conidia of the $\Delta U v m s n 2$ mutant were abnormal; they were larger or longer in size than the wild-type and complemented strains (Fig. 2c, d). Additionally, we also evaluated conidial germination, and found that the vast majority of conidia formed one germ tube in the $\Delta U v m s n 2$ mutant, whereas multiple germ tubes in the wild-type and complemented strains (Fig. 3). The results suggest that UvMsn2 plays an important role in vegetative growth, conidiation, and also conidial morphology and germination in $U$. virens.

\section{UvMsn2 plays a crucial role in response of $U$. virens to environmental stresses}

To determine whether UvMsn2 is involved in response of $U$. virens to environmental stresses, HWD-2, $\Delta U v m s n 2$ and $\Delta U v m s n 2 / U v M S N 2$ were inoculated onto YT plates supplemented with different stress agents, including $\mathrm{NaCl}$ and sorbitol (osmotic stresses), $\mathrm{H}_{2} \mathrm{O}_{2}$ (oxidative stress), and SDS, CFW and CR (cell wall stresses). After incubation at $25^{\circ} \mathrm{C}$ for 14 days, the $\Delta U v m s n 2 \mathrm{mu}$ tant showed a decreased tolerance to sorbitol, $\mathrm{H}_{2} \mathrm{O}_{2}$ and SDS, and an increased tolerance to CFW and CR, and exhibited no remarkable difference to $\mathrm{NaCl}$ in comparison to the wild-type HWD-2 and complemented strain $\triangle U v m s n 2 / U_{v M S N 2}$ (Fig. 4a, b). The results indicate that UvMsn2 plays a crucial role in response to environmental stresses in $U$. virens. 

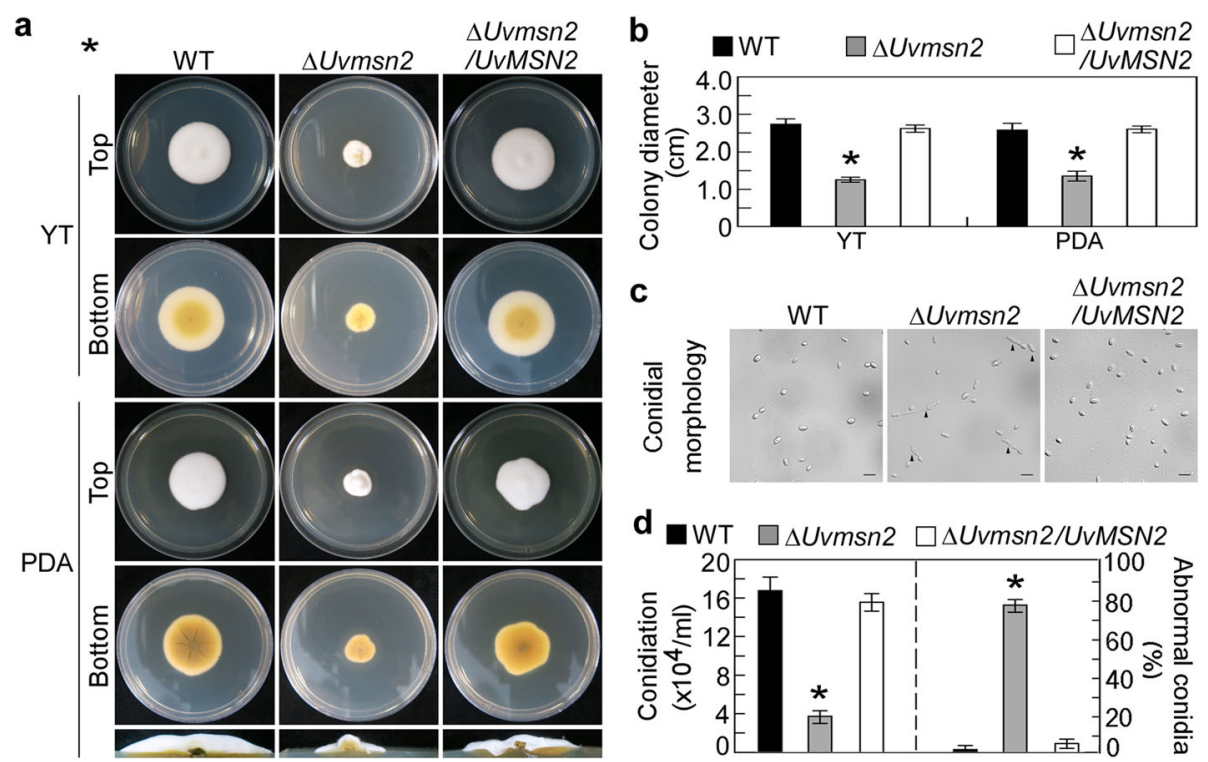

Fig. 2 UVMsn2 is important for vegetative growth and conidiogenesis in Ustilaginoidea virens. a Vegetative growth and colony morphology of the wild-type HWD-2, $\Delta$ UVmsn2 mutant and the complemented transformant $\triangle$ UVmsn2/UVMSN2 cultured on YT and PDA agar plates, and photographed at $14 \mathrm{dpi}$ at $25^{\circ} \mathrm{C}$ in the dark. $\mathbf{b}$ Statistical analysis of the colony diameter of the indicated strains. The mean values $\pm S D$ were calculated from three independent experiments, and asterisks indicate a statistically significant difference from the wild-type strain $(P<0.01)$. $\mathbf{C}$ Conidial morphology of the indicated strains. Black triangles indicate abnormal conidia. Bar $=10 \mu \mathrm{m}$. $\mathbf{d}$ Conidial production of the indicated strains. The mean values \pm SD were calculated from three independent experiments, and asterisks indicate a statistically significant difference from the wild-type strain $(P<0.01)$

\section{UvMsn2 is important for pathogenicity and production of} phytotoxic compounds in $U$. virens

To determine the role of UvMsn2 in virulence of $U$. virens, we inoculated conidial suspensions of HWD-2, $\Delta U \nu m s n 2$ and $\Delta U \nu m s n 2 / U v M S N 2$ into rice panicles by injection method. At 4 weeks post-inoculation, the $\Delta U \nu m s n 2$ mutant caused no symptoms on grains, and no false smut balls were observed on rice panicles. In contrast, the wild-type HWD-2 and complemented strain $\Delta U v m s n 2 / U v M S N 2$ caused severe symptoms, with over five false smut balls per rice panicle (Fig. 5a). We further investigated the role of UvMsn2 in regulating the production of phytotoxic compounds by harvesting 5day-old YT culture filtrates of HWD-2, $\Delta U v m s n 2$ and $\triangle U \nu m s n 2 / U v M S N 2$ strains, and subjecting them to rice seed germination assays. The results showed that rice seeds germinated much longer shoots (2.4-fold) in $\Delta U \nu m s n 2$-treated group than in wild-type and complemented strains-treated groups (Fig. 5b, c). Additionally, we analyzed the expression levels of five ustilaginoidin biosynthetic genes, i.e., USTA (the precursor protein encoding gene for ustiloxins) (Tsukui et al. 2015), PKS1

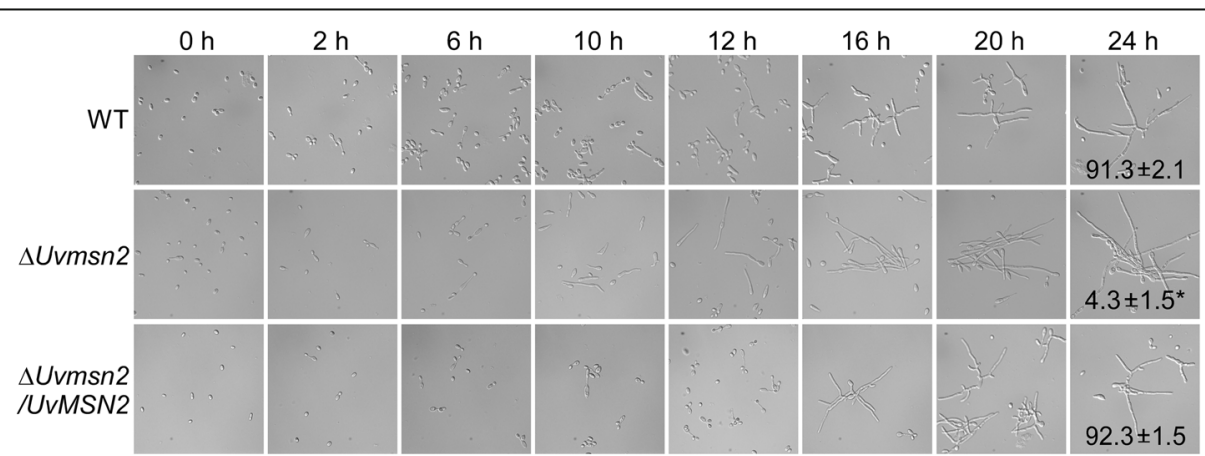

Fig. 3 UvMsn2 is important for conidial germination in Ustilaginoidea virens. Conidia of the wild-type HWD-2, $\Delta U v m s n 2$ and $\Delta U v m s n 2 / U v M S N 2$ strains were incubated in liquid YTS medium. Examination of conidial germination under a microscopy was performed at 0, 2, 6, 10, 12, 16, 20 and $24 \mathrm{~h}$ after incubation. The number at the last column represents the percentage of multiple germ tubes of the indicated strains at $24 \mathrm{~h}$ after incubation. The mean values \pm SD were calculated from three independent experiments, and asterisk indicates a statistically significant difference from the wild-type strain $(P<0.01)$ 


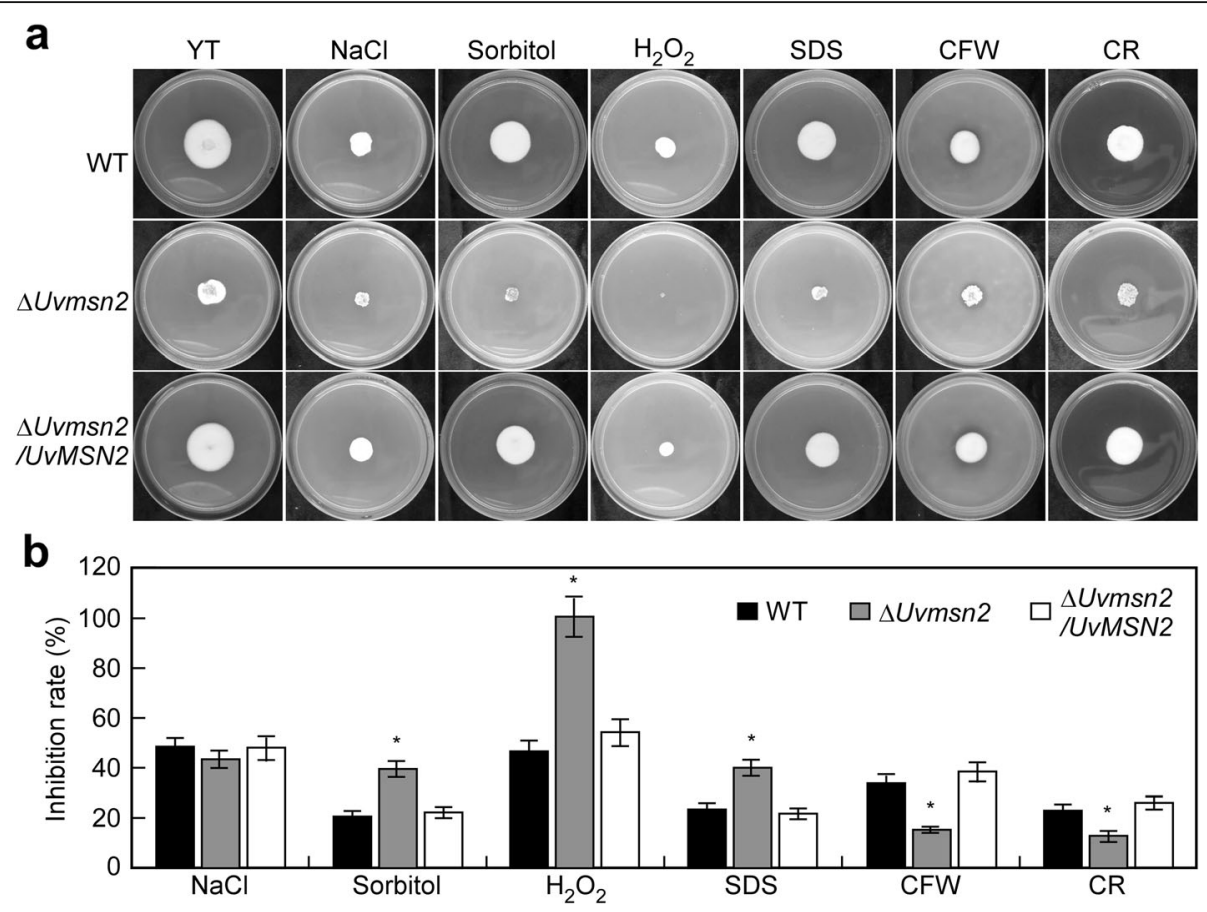

Fig. 4 UVMsn2 plays a crucial role in regulating stress responses in Ustilaginoidea virens. a The wild-type HWD-2, $\Delta U v m s n 2$ and $\Delta U v m s n 2 / U v M S N 2$ strains were incubated on YT agar plates supplemented with different stress agents at $25^{\circ} \mathrm{C}$ for 14 days. b Statistical analysis of the growth inhibition rate of the indicated strains exposed to different stresses. The mean values \pm SD were calculated from three independent experiments, and asterisks indicate a statistically significant difference from the wild-type strain $(P<0.01)$
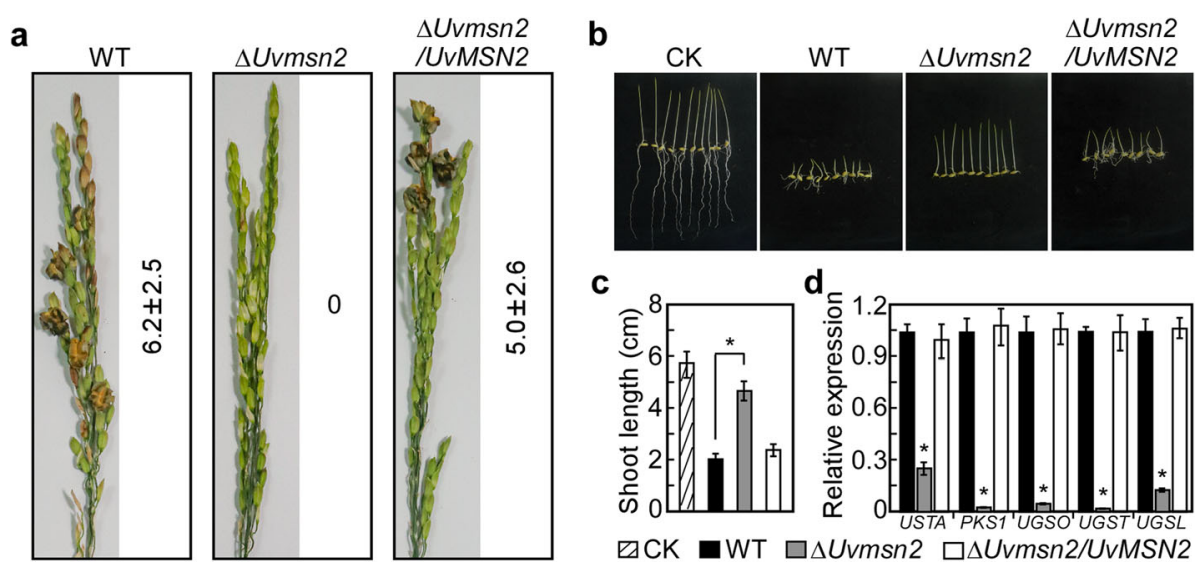

Fig. 5 UvMsn2 is essential for pathogenicity of Ustilaginoidea virens. a Conidial suspensions collected from the wild-type HWD-2, $\triangle U v m s n 2$ and $\triangle$ UVmsn2/UVMSN2 strains were inoculated into rice panicles and photographed at $28 \mathrm{dpi}$. The number of false smut balls on each panicle was counted and analyzed. $\mathbf{b}$ Seeds of rice cultivar were incubated on filter papers soaked with blank control (liquid YT medium) or filtrates of 5-day-old YT cultures of the indicated strains. Shoot length was examined after incubation at $25^{\circ} \mathrm{C}$ for 5 days. c Statistical analysis of the shoot length of the indicated strains. The mean values \pm SD were calculated from three independent experiments, and asterisk indicates a statistically significant difference from the wild type $(P<0.01)$. d RT-qPCR analyses of the expression of ustilaginoidin biosynthetic genes in the indicated strains. The mean values \pm SD were calculated from three independent experiments, and asterisk indicates a statistically significant difference from the wild-type strain $(P<0.01)$ 
(polyketide synthase gene), UGSO, UGST and UGSL (major facilitator superfamily transporter encoding genes required for the modification of the primary PKS products) (Li et al. 2019), and found that all these five genes were significantly downregulated in the $\Delta U v m s n 2 \mathrm{mu}-$ tant (Fig. 5d). The results suggest that UvMsn2 is important for pathogenicity and production of phytotoxic compounds in $U$. virens.

UvMsn2 is required for the maintenance of mitochondrial morphology in $U$. virens

Msn2 is involved in virulence by regulating mitochondrial morphology in M. oryzae (Xiao et al. 2021). To determine whether Msn2 has a similar role in $U$. virens, we compared the mitochondrial morphology of HWD-2, $\Delta U v m s n 2$ and $\Delta U v m s n 2 / U v M S N 2$ strains in hyphae and conidia stained with Mitotracker Red. The results revealed that $80 \%$ mitochondria were tubular in both hyphae and conidia of the wild-type and complemented strains. In contrast, $80 \%$ mitochondria were punctate in the mutant. When treated with Mdivi-1, a mitochondrial fission inhibitor, $70 \%$ mitochondria of the $\Delta U v m s n 2 \mathrm{mu}-$ tant were restored to tubular morphology (Fig. 6a, b). The results indicate that UvMsn2 is essential for the maintenance of mitochondrial morphology.

\section{Subcellular localization of UvMsn2 in U. virens}

To determine the localization pattern of UvMsn2 in $U$. virens, we examined the hyphae and conidia of the complemented strain $\triangle U v m s n 2 / U v M S N 2$ under a confocal fluorescence microscope. We found that strong GFP signals were accumulated in punctate structures, and relative weak GFP signals were distributed in cytosol in both hyphae and conidia. We then stained the hyphae and conidia with DAPI (fluorescent dye that binds to DNA) and found GFP signals were merged well with DAPI signals (Fig. 7a). These findings indicate that UvMsn2 is localized in both cytosol and nucleus in $U$. virens. We further observed the localization pattern of UvMsn2 under the treatment of $\mathrm{NaCl}$, and found that UvMsn2-GFP could translocate from cytosol to nucleus (Fig. 7b), which is similar to MoMsn2 in M. oryzae.

\section{Discussion}

In this study, we identified a zinc finger TF UvMsn2 in the rice false smut fungus $U$. virens, and found that UvMsn2 showed the conserved domain in protein sequence and similar biological functions to MoMsn2 from $M$. oryzae, indicating that Msn2 is likely a wellconserved protein across different phytopathogens.

Transcription regulation mediated by TFs is critical for driving multiple cellular activities. A large number of TFs have been characterized to play crucial roles in growth, development, stress response and infection of $M$. oryzae by directly or indirectly regulating the expression of downstream genes ( $\mathrm{Lu}$ et al. 2014; Kong et al. 2015; Tang et al. 2015; Cao et al. 2016). In $U$. virens, many TFs such as GATA-binding TF family and basic leucine zipper (bZIP) TF family have been genomewidely identified (Yin et al. 2017; Yu et al. 2019), but their biological functions are still not studied in detail. Only several TFs (UvHox2, UvPro1 and UvCom1) are well characterized to play important roles in vegetative growth, conidiation, stress response and plant infection

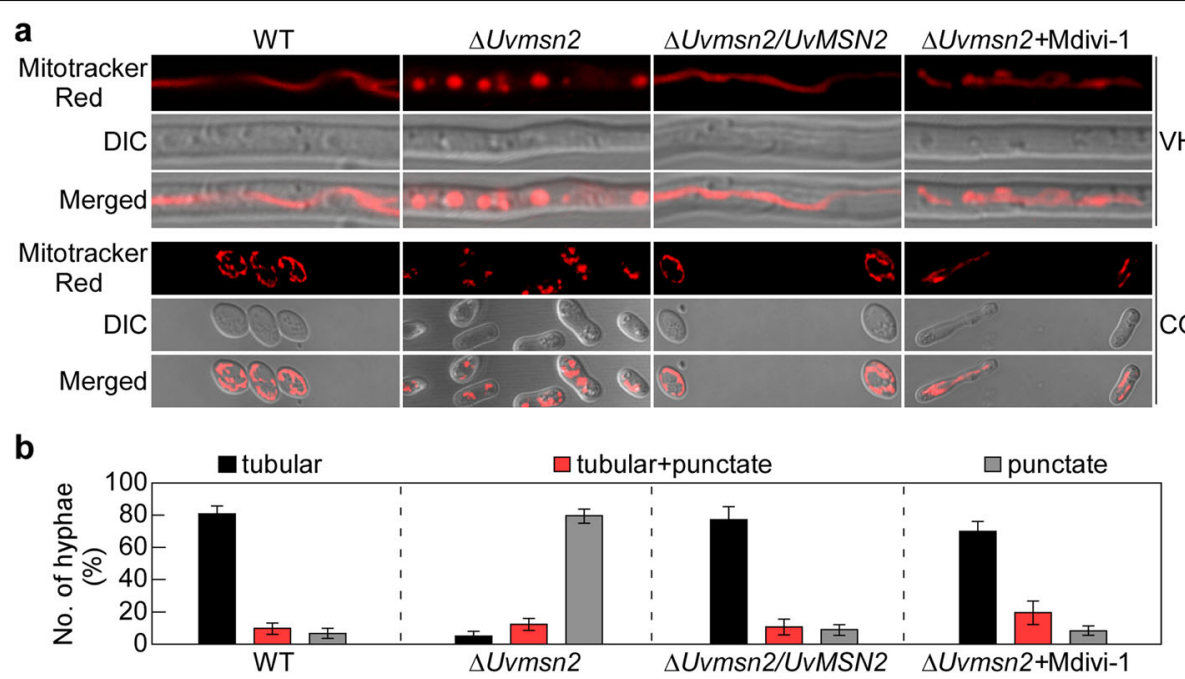

Fig. 6 UvMsn2 is important for the maintenance of mitochondrial morphology in Ustilaginoidea virens. a Confocal microscopic observation of mitochondria in hyphae and conidia of HWD-2, $\Delta$ UVmsn2 and $\Delta$ UVmsn2/UVMSN2 treated or untreated with Mdivi-1 and stained with Mitotracker Red. VH, vegetative hyphae; CO, conidia. $\mathbf{b}$ Statistical analysis of the mitochondrial morphology in the indicated strains. The mean values \pm SD were calculated from three independent experiments 

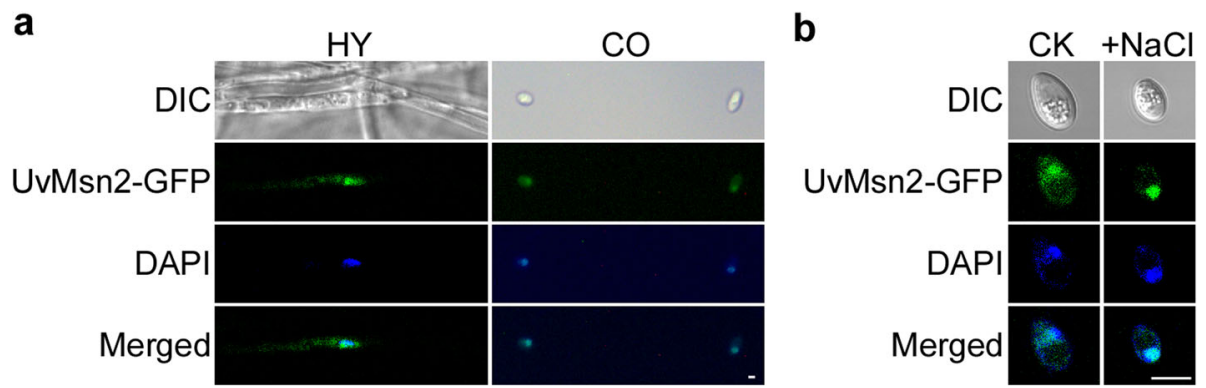

Fig. 7 Subcellular localization of UvMsn2 in Ustilaginoidea virens. a Hyphae and conidia expressing UvMsn2-GFP was stained with DAPI and examined under a confocal microscope. $\mathbf{b}$ Epifluorescence observation of the conidia untreated (ck) or treated with $0.4 \mathrm{M} \mathrm{NaCl}$ for $30 \mathrm{~min}$. DAPI was added to the cultures 5 min before observation. Bar $=5 \mu \mathrm{m}$

(Lv et al. 2016; Zheng et al. 2017; Chen et al. 2020b). Here, we found that UvMsn2 is a key TF that regulates the development and virulence of $U$. virens.

One of the important and conserved functions of Msn2 in yeast and fungi is its involvement in stress response (Gasch et al. 2000). In budding yeast, Msn2 is involved in responses to various stresses, including salt, osmotic, oxidative and carbon starvation stresses; it is translocated from cytosol to nucleus under stress conditions (Gorner et al. 1998; Boisnard et al. 2009; Rajvanshi et al. 2017). In two entomopathogenic fungi, Msn2 is required for multi-stress responses, including hyperosmolarity, oxidation, carbendazim, cell wall perturbing and high temperature stress (Liu et al. 2013). In M. oryzae, MoMsn2 is also involved in response to hyperosmolarity and cell wall perturbing stresses, and is translocated from cytosol to nucleus under $\mathrm{NaCl}$ treatment (Zhang et al. 2014). In consistent with these findings, we found that UvMsn2 also played important roles in multi-stress response of $U$. virens. Meanwhile, both $\Delta U v m s n 2$ and $\triangle M o m s n 2$ mutants were more tolerant to CFW and CR, which is similar to some yeast mutants (Imai et al. 2005). One possible explanation is that the chitin level in cell wall is not the only factor determining CFW and CR sensitivity (Ram and Klis 2006). However, Msn2and Msn4-like TFs have no obvious roles in stress responses in the fungal pathogen Candida albicans (Nicholls et al. 2004). These findings indicate that Msn2 is a key TF involved in stress response, though it exhibits diverged role during stress signaling in some fungi.

In addition to stress response, Msn2/4 plays very important roles in regulating infection-related morphogenesis in fungal pathogens, such as conidiation and virulence (Liu et al. 2013; Zhang et al. 2014). In M. ory$z a e$, we found that MoMsn2 regulates vegetative growth, conidial production and morphology, mitochondrial morphology and infection by directly or indirectly regulating the expression of downstream genes (Zhang et al. 2014). Similar results were obtained for UvMsn2, and it is also important for vegetative growth, conidiogenesis, mitochondrial morphology and pathogenicity in $U$. virens. Meanwhile, UvMsn2 showed a similar localization pattern with MoMsn2; it is localized in both the cytosol and nucleus, and could also be translocated from cytosol to nucleus under stress treatment. All these findings indicate that UvMsn2 and MoMsn2 have a similar biological role, further supporting the result that UvMsn2 is the homolog of MoMsn2. In addition, we found that targeted-deletion of $U v M s n 2$ in $U$. virens resulted in less toxicity of its culture filtrates on rice seeds and the expression of several ustilaginoidin biosynthetic related genes was significantly decreased. Toxins are virulence factor contributing to infection of host plants by some pathogens (Proctor et al. 1995; Yang et al. 2016; Yang et al. 2017). Therefore, we suppose that UvMsn2 is likely involved in toxin production, thus contributing to the virulence of $U$. virens. However, the underlying mechanisms on how UvMsn2 regulates these processes need to be further explored.

\section{Conclusions}

In this study, we identified and characterized a zinc finger TF, UvMsn2, in $U$. virens, and found that UvMsn2 is important for vegetative growth, conidiogenesis, stress response, mitochondrial morphology and pathogenicity of this fungal pathogen. These findings provide new evidences to better understand the pathogenic mechanisms of $U$. virens.

\section{Methods}

\section{Fungal strains and culture conditions}

All of the $U$. virens strains were cultured on PSA (200 g of peeled potato, $20 \mathrm{~g}$ of sucrose, and $15 \mathrm{~g}$ of agar in $1 \mathrm{~L}$ of distilled water) in the dark at $25^{\circ} \mathrm{C}$. For conidiation, mycelial blocks were cultured in PSB $(200 \mathrm{~g}$ of peeled potato, $20 \mathrm{~g}$ of sucrose in $1 \mathrm{~L}$ of distilled water) by shaking at $145 \mathrm{rpm}, 28^{\circ} \mathrm{C}$ for 7 days. For vegetative growth, $2 \mathrm{~mm} \times 2 \mathrm{~mm}$ mycelial blocks from freshly cultured strains were inoculated onto potato dextrose agar (PDA) or YT $(0.1 \%$ yeast extract, $0.1 \%$ tryptone, and $1 \%$ 
glucose) (Tanaka et al. 2011) plates, then cultured in the dark at $25^{\circ} \mathrm{C}$ for 14 days. To test sensitivity against different stresses, vegetative growth was assayed after incubation at $25^{\circ} \mathrm{C}$ for 14 days on regular YT plates and YT with $0.4 \mathrm{M} \mathrm{NaCl}, 0.5 \mathrm{M}$ sorbitol, $0.07 \% \mathrm{H}_{2} \mathrm{O}_{2}, 0.03 \%$ SDS (w/v), $70 \mu \mathrm{g} / \mathrm{mL}$ Congo red (CR) or $100 \mu \mathrm{g} / \mathrm{mL}$ calcofluor white (CFW). Freshly harvested conidia were resuspended with YTS $(0.1 \%$ yeast extract, $0.1 \%$ tryptone, and $1 \%$ sucrose) to a concentration of $1 \times 10^{6}$ conidia/ $\mathrm{mL}$, which was used to assay conidial germination after incubated for $0,2,6,10,12,16,20$ and $24 \mathrm{~h}$. The wildtype strain HWD-2 (Chen et al. 2020a) was obtained from Dr. Lu Zheng's Lab, Huazhong Agriculture University. All $M$. oryzae strains were cultured on complete medium $(\mathrm{CM})$ at $28^{\circ} \mathrm{C}$ in the dark. For vegetative growth, $2 \mathrm{~mm} \times 2 \mathrm{~mm}$ mycelial blocks from freshly cultured strains were inoculated into CM plates and cultured at $28^{\circ} \mathrm{C}$ for 7 days in the dark. For conidial production, strains were inoculated onto straw decoction and corn powder plates (SDC, $100 \mathrm{~g}$ straw, $40 \mathrm{~g}$ corn powder, $15 \mathrm{~g}$ agar in $1 \mathrm{~L}$ of distilled water) for 7 days in the dark followed by 3 days of continuous illumination under fluorescent light as described (Li et al. 2016). The $M$. oryzae Guy11 strain was used as wild type in this study, the $\Delta M o m s n 2$ mutant was acquired in our previous study (Zhang et al. 2014).

\section{Heterogenous complementation assay}

For heterogenous complementation the $\Delta M o m s n 2 \mathrm{mu}-$ tant, the entire $U v M S N 2$ gene including its $1.5-\mathrm{kb}$ promoter region was amplified by PCR, and cloned into the pYF11 plasmid (bleomycin resistant) digested with XhoI as described (Bruno et al. 2004). The resulting pYF11UvMSN2-GFP construct was transformed into the protoplast of $\triangle M o m s n 2$. The bleomycin-resistant transformants with GFP signals were selected for phenotype analysis.

\section{Construction of the replacement vector and Cas9-gRNA vector for targeted-deletion of UVMSN2}

To generate the $U v M S N 2$ gene replacement vector pMD19-T-UvMSN2KO, the $1 \mathrm{~kb}$ upstream and $1 \mathrm{~kb}$ downstream flanking sequences of $U v M S N 2$ were amplified with primers $1 F / 2 R$ and $3 F / 4 R$ (Additional file 2: Table S1), and cloned into the pMD19-T vector as described previously (Zhang et al. 2010). To generate Cas9-gRNA vector pCas9-tRp-gRNA-UvMSN2, the gRNA spacers were designed with the gRNA designer program (http://grna.ctegd.uga.edu/), and cloned into the pCas9-tRp-gRNA vector as described (Arazoe et al. 2015; Guo et al. 2019).

UvMSN2 gene deletion and complementation The resulting pMD19-T-UvMSN2KO and pCas9-tRpgRNA-UvMSN2 vectors were co-transformed into protoplasts of the wild-type strain HWD-2 according to the Magnaporthe transformation approach to obtain the gene deletion mutants (Talbot et al. 1993). Hygromycin-resistant transformants were screened by PCR, and confirmed by southern blot analysis. For complementation, the above pYF11-UvMSN2-GFP construct was transformed into the protoplast of $\Delta U v m s n 2$. The bleomycin-resistant transformants with GFP signals were selected for phenotype analysis.

\section{Pathogenicity assay of $M$. oryzae strains}

The 2-week-old seedlings of susceptible rice cultivar CO-39 and 7-day-old barley (cv. Four-arris) were used for inoculation assays as described previously (Zhang et al. 2014). Mycelial plugs of the wild-type Guy11, $\Delta$ Momsn2 mutant and heterologous complemented transformant $\triangle M o m s n 2 / U v M S N 2$ were inoculated onto detached rice and barely leaves, which were then placed in a growth chamber at $28{ }^{\circ} \mathrm{C}$ with $90 \%$ humidity and in the dark for the first $24 \mathrm{~h}$, followed by a $12 \mathrm{~h} / 12 \mathrm{~h}$ light/ dark cycle. Diseased leaves were photographed at 5 and 7 days post-inoculation (dpi) for barley and rice, respectively.

\section{Toxicity assays with culture filtrates}

Toxicity assays with culture filtrates were performed according to the method described previously (Zheng et al. 2016). Mycelial blocks $(2 \mathrm{~mm} \times 2 \mathrm{~mm})$ were cut from the edge of 14-day old strains and cultured in liquid YT medium by shaking for 5 days. The mycelia were collected by filtration through two layers of Miracloth (EMD Millipore Corporation, Billerica, MA 01821 USA) and grinded into powder, and the dry weight was measured after lyophilizing for $24 \mathrm{~h}$. The culture filtrates were then centrifuged at $3500 \mathrm{rpm}$ for $8 \mathrm{~min}$ to collect the supernatants. Seeds of rice cultivar CO-39 were incubated on sterile filter papers soaked with the culture filtrates at $25^{\circ} \mathrm{C}$. The shoot and root growth were measured after incubation for 5 days.

\section{Reverse transcription quantitative PCR (RT-qPCR) assay}

Total RNA samples were extracted from freshly prepared mycelia using Pure linkTM RNA mini kit (Invitrogen). The cDNA was prepared using reverse transcriptase HiScript III RT SuperMix for qPCR (Vazyme Biotech Co., Nanjing, China). qPCR was run on an Applied Biosystems (Foster City, CA, USA) 7500 Real Time PCR System with SYBR Premix ExTaq (Vazyme Biotech Co., Nanjing, China). The relative quantification of each transcript was calculated by the $2^{-\Delta \Delta C T}$ method (Livak and Schmittgen 2001) with the $U$. virens ACTIN gene (UV_6104) as the internal control. The experiment was repeated three times with three biological replicates each time. 
Pathogenicity assay of $U$. virens strains on rice panicles Inoculation suspensions were prepared as described previously (Guo et al. 2019). The spore and mycelium mixture were broken into pieces and adjusted to a concentration of $3 \times 10^{6}$ spores $/ \mathrm{mL}$. One milliliter of suspensions was inoculated onto each spike and ten spikes for each strain as described previously (Zheng et al. 2017). The inoculated plants were kept in a chamber at $25^{\circ} \mathrm{C}$ for 28 days with $>70 \%$ humidity.

\section{Microscopic observation}

The complemented transformant expressing $U v M S N 2::$ GFP was used for subcellular localization observation. Hyphae and conidia were stained with the nucleusspecific dye 4',6-diamidino-2-phenylindole (DAPI, Sigma-Aldrich, D8417) at a concentration of $10 \mu \mathrm{g} / \mathrm{mL}$ at room temperature for $3-5 \mathrm{~min}$ in darkness, then examined under a confocal fluorescence microscope (LSM710, 63×oil; Zeiss). For mitochondrial staining, hyphae and conidia of the indicated strains treated or untreated with Mdivi-1, were stained by the mitochondriaspecific marker Mitotracker Red CMXRos (Invitrogen, Cat. M7512) at a concentration of $100 \mathrm{nM}$ at room temperature for 1-2 min in darkness, and observed under a confocal microscope (LSM710, $63 \times$ oil; Zeiss).

\section{Abbreviations}

aa: Amino acid; CFW: Calcofluor white; CR: Congo red; CRISPR: Clustered regularly interspaced short palindromic repeats; DAPI: 4',6-diamidino-2phenylindole; GFP: Green fluorescent protein; HPH: Hygromycin B phosphotransferase; NLS: Nuclear localization signal sequence; TF: Transcription factor; TOR: Target of rapamycin; PCR: Polymerase chain reaction; SDS: Sodium dodecyl sulfate

\section{Supplementary Information}

The online version contains supplementary material available at https://doi. org/10.1186/s42483-021-00093-y.

Additional file 1: Figure S1. UvMsn2 rescues the defect in mitochondrial morphology of the $\Delta$ Momsn2 mutant. Mitochondria in hyphae of the Magnaporthe oryzae wild-type Guy11, Momsn2 mutant and heterologous complemented transformant Momsn2/UVMSN2 were stained with Mitotracker Red and examined under a confocal microscope. Figure S2. Targeted-deletion of UVMSN2 in Ustilaginoidea virens. a Schematic diagram showing strategy for deletion of UVMSN2 from the genome of $U$. virens. $\mathbf{b}$ Southern blot analysis of the gene knockout mutant using probe 1 and probe 2 corresponding to UVMSN2 and HPH, respectively.

Additional file 2: Table S1. Primers used in this study.

\section{Acknowledgements}

We thank Prof. Jin-Rong Xu at Purdue University for providing the pCas9tRpgRNA vector. We also thank Dr. Lu Zheng at Huazhong Agricultural University for providing the U. virens field isolate HWD-2.

\section{Authors' contributions}

$Y X, Z Z$ and $H Z$ designed the research; YX, SW, ZMY, EKM and ZXY performed the experiments; $Y X, S W, Z M Y$ and $H Z$ analyzed the data; and $Y X, S W, Z Z$ and $\mathrm{HZ}$ wrote the manuscript. All authors read and approved the final manuscript.

\section{Funding}

This study was supported by the National Key Research and Development Program of China (Grant No: 2016YFD0300700/2016YFD0300706), the Natural Science Foundation of China (Grant No: 32061143045 to H. Zhang), the Fundamental Research Funds for the Central Universities (Grant No: KYYZ201903 to H. Zhang).

Availability of data and materials

Not applicable.

\section{Declarations}

Ethics approval and consent to participate

Not applicable.

Consent for publication

Not applicable.

\section{Competing interests}

The authors declare that they have no competing interests.

Received: 22 April 2021 Accepted: 7 July 2021

Published online: 31 July 2021

\section{References}

Arazoe T, Miyoshi K, Yamato T, Ogawa T, Ohsato S, Arie T, et al. Tailor-made CRIS $\mathrm{PR} /$ Cas system for highly efficient targeted gene replacement in the rice blast fungus. Biotechnol Bioeng. 2015;112(12):2543-9. https://doi.org/10.1 002/bit.25662.

Boisnard S, Lagniel G, Garmendia-Torres C, Molin M, Boy-Marcotte E, Jacquet M et al. $\mathrm{H}_{2} \mathrm{O}_{2}$ activates the nuclear localization of Msn2 and Maf1 through thioredoxins in Saccharomyces cerevisiae. Eukaryot Cell. 2009;8(9):1429-38. https://doi.org/10.1128/EC.00106-09.

Bruno KS, Tenjo F, Li L, Hamer JE, XU JR. Cellular localization and role of kinase activity of PMK1 in Magnaporthe grisea. Eukaryot Cell. 2004;3(6):1525-32. https://doi.org/10.1128/EC.3.6.1525-1532.2004.

Cao H, Huang P, Zhang L, Shi Y, Sun D, Yan Y, et al. Characterization of 47 Cys2His2 zinc finger proteins required for the development and pathogenicity of the rice blast fungus Magnaporthe oryzae. New Phytol. 2016;211(3):1035-51. https://doi.org/10.1111/nph.13948.

Chen X, Hai D, Tang J, Liu H, Huang J, Luo C, et al. UvCom1 is an important regulator required for development and infection in the rice false smut fungus Ustilaginoidea virens. Phytopathology. 2020b;110(2):483-93. https:// doi.org/10.1094/PHYTO-05-19-0179-R.

Chen X, Tang J, Pei Z, Liu H, Huang J, Luo C, et al. The 'pears and lemons' protein UvPal1 regulates development and virulence of Ustilaginoidea virens. Environ Microbiol. 2020a;22(12):5414-32. https://doi.org/10.1111/1462-2920.15284.

Crespo JL, Hall MN. Elucidating TOR signaling and rapamycin action: lessons from Saccharomyces cerevisiae. Microbiol Mol Biol Rev. 2002;66(4):579-91. https:// doi.org/10.1128/MMBR.66.4.579-591.2002.

Estruch F, Carlson M. Two homologous zinc-finger genes identified by multicopy suppression in a Snf1 protein kinase mutant of Saccharomyces cerevisiae. Mol Cell Biol. 1993;13(7):3872-81. https://doi.org/10.1128/ mcb.13.7.3872-3881.1993

Gasch AP, Spellman PT, Kao CM, Carmel-Harel O, Eisen MB, Storz G, et al. Genomic expression programs in the response of yeast cells to environmental changes. Mol Biol Cell. 2000;11(12):4241-57. https://doi.org/1 0.1091/mbc.11.12.4241.

Gorner W, Durchschlag E, Martinez-Pastor MT, Estruch F, Ammerer G, Hamilton B, et al. Nuclear localization of the $\mathrm{C}_{2} \mathrm{H}_{2}$ zinc finger protein Msn2p is regulated by stress and protein kinase a activity. Genes Dev. 1998;12(4):586-97. https:// doi.org/10.1101/gad.12.4.586.

Gorner W, Durchschlag E, Wolf J, Brown EL, Ammerer G, Ruis H, et al. Acute glucose starvation activates the nuclear localization signal of a stress-specific yeast transcription factor. EMBO J. 2002;21(1):135-44. https://doi.org/10.1093/ emboj/21.1.135.

Guo WW, Gao YX, Yu ZM, Xiao YH, Zhang ZG, Zhang HF. The adenylate cyclase UVAC1 and phosphodiesterase UVPdeH control the intracellular cAMP level, development, and pathogenicity of the rice false smut fungus Ustilaginoidea virens. Fungal Genet Biol. 2019;129:65-73. https:// doi.org/10.1016/j.fgb.2019.04.017. 
Imai K, Noda Y, Adachi H, Yoda K. A novel endoplasmic reticulum membrane protein Rcr1 regulates chitin deposition in the cell wall of Saccharomyces cerevisiae. J Biol Chem. 2005;280(9):8275-84. https://doi.org/10.1074/jbc.M4 09428200.

Kong S, Park SY, Lee YH. Systematic characterization of the bZIP transcription factor gene family in the rice blast fungus, Magnaporthe oryzae. Environ Microbiol. 2015;17(4):1425-43. https://doi.org/10.1111/1462-2920.12633.

Li M, Liu X, Liu Z, Sun Y, Liu M, Wang X, et al. Glycoside hydrolase MoGls2 controls asexual/sexual development, cell wall integrity and infectious growth in the rice blast fungus. PLoS One. 2016;11(9):e0162243. https://doi. org/10.1371/journal.pone.0162243.

Li YJ, Wang M, Liu ZH, Zhang K, Cui FH, Sun WX. Towards understanding the biosynthetic pathway for ustilaginoidin mycotoxins in Ustilaginoided virens. Environ Microbiol. 2019;21(8):2629-43. https://doi.org/10.1111/14 62-2920.14572.

Liang YF, Han Y, Wang CF, Jiang C, Xu JR. Targeted deletion of the USTA and UVSLT2 genes efficiently in Ustilaginoidea virens with the CRISPR-Cas9 system. Front Plant Sci. 2018;9:699. https://doi.org/10.3389/fpls.2018.00699.

Liu Q, Ying SH, Li JG, Tian CG, Feng MG. Insight into the transcriptional regulation of $\mathrm{Msn} 2$ required for conidiation, multi-stress responses and virulence of two entomopathogenic fungi. Fungal Genet Biol. 2013;54:42-51. https://doi.org/10.1016/j.fgb.2013.02.008.

Livak KJ, Schmittgen TD. Analysis of relative gene expression data using real-time quantitative PCR and the 2(-Delta Delta C(T)) method. Methods. 2001;25(4): 402-8. https://doi.org/10.1006/meth.2001.1262.

Lu J, Cao H, Zhang L, Huang P, Lin F. Systematic analysis of Zn2Cys6 transcription factors required for development and pathogenicity by high-throughput gene knockout in the rice blast fungus. PLoS Pathog. 2014;10(10):e1004432. https://doi.org/10.1371/journal.ppat.1004432.

Lv B, Zheng L, Liu H, Tang JT, Hsiang T, Huang JB. Use of random T-DNA mutagenesis in identification of gene UVPRO1, a regulator of conidiation, stress response, and virulence in Ustilaginoidea virens. Front Microbiol. 2016;7:2086.

Nicholls S, Straffon M, Enjalbert B, Nantel A, Macaskill S, Whiteway M, et al. Msn2and Msn4-like transcription factors play no obvious roles in the stress responses of the fungal pathogen Candida albicans. Eukaryot Cell. 2004;3(5): 1111-23. https://doi.org/10.1128/EC.3.5.1111-1123.2004.

Proctor RH, Hohn TM, McCormick SP. Reduced virulence of Gibberella zeae caused by disruption of a trichothecene toxin biosynthetic gene. Mol PlantMicrobe Interact. 1995;8(4):593-601. https://doi.org/10.1094/MPMI-8-0593.

Rajvanshi PK, Arya M, Rajasekharan R. The stress-regulatory transcription factors Msn2 and Msn4 regulate fatty acid oxidation in budding yeast. J Biol Chem. 2017;292(45):18628-43. https://doi.org/10.1074/jbc.M117.801704.

Ram AFJ, Klis FM. Identification of fungal cell wall mutants using susceptibility assays based on Calcofluor white and Congo red. Nat Protoc. 2006;1(5):22536. https://doi.org/10.1038/nprot.2006.397.

Stewart-Ornstein J, Nelson C, DeRisi J, Weissman JS, El-Samad H. Msn2 coordinates a stoichiometric gene expression program. Curr Biol. 2013;23(23): 2336-45. https://doi.org/10.1016/j.cub.2013.09.043.

Sun W, Fan J, Fang AF, Li YJ, Tariqjaveed M, Li DY, et al. Ustilaginoidea virens: insights into an emerging rice pathogen. Annu Rev Phytopathol. 2020;58(1): 363-85. https://doi.org/10.1146/annurev-phyto-010820-012908.

Talbot NJ, Ebbole DJ, Hamer JE. Identification and characterization of MPG1, a gene involved in pathogenicity from the rice blast fungus Magnaporthe grisea. Plant Cell. 1993:5(11):1575-90. https://doi.org/10.1105/tpc.5.11.1575.

Tanaka E, Kumagawa T, Tanaka C, Koga H. Simple transformation of the rice false smut fungus Villosiclava virens by electroporation of intact conidia. Mycoscience. 2011;52(5):344-8. https://doi.org/10.1007/S10267-011-0115-6.

Tang W, Ru YY, Hong L, Zhu Q, Zuo RF, Guo XX, et al. System-wide characterization of bZIP transcription factor proteins involved in infectionrelated morphogenesis of Magnaporthe oryzae. Environ Microbiol. 2015;17(4): 1377-96. https://doi.org/10.1111/1462-2920.12618.

Tsukui T, Nagano N, Umemura M, Kumagai T, Terai G, Machida M, et al. Ustiloxins, fungal cyclic peptides, are ribosomally synthesized in Ustilaginoidea virens. Bioinformatics. 2015;31(7):981-5. https://doi.org/10.1093/bioinformatics/ btu753.

Xiao YH, Liu LP, Zhang T, Zhou RW, Ren Y, Li XR, et al. Transcription factor MoMsn2 targets the putative 3-methylglutaconyl-CoA hydratase-encoding gene MoAUH1 to govern infectious growth via mitochondrial fusion/fission balance in Magnaporthe oryzae. Environ Microbiol. 2021;23(2):774-90. https:// doi.org/10.1111/1462-2920.15088.
Yang KL, Liu YH, Liang LL, Li ZG, Qin QP, Nie XY, et al. The high-affinity phosphodiesterase $\mathrm{PdeH}$ regulates development and aflatoxin biosynthesis in Aspergillus flavus. Fungal Genet Biol. 2017;101:7-19. https://doi.org/10.101 6/j.fgb.2017.02.004.

Yang KL, Qin QP, Liu YH, Zhang LM, Liang LL, Lan HH, et al. Adenylate cyclase AcyA regulates development, aflatoxin biosynthesis and fungal virulence in Aspergillus flavus. Front Cell Infect Microbiol. 2016;6:190.

Yin WX, Cui P, Wei W, Lin Y, Luo CX. Genome-wide identification and analysis of the basic leucine zipper (bZIP) transcription factor gene family in Ustilaginoidea virens. Genome. 2017;60:1059-67.

Yu M, Yu JJ, Cao HJ, Yong ML, Liu YF. Genome-wide identification and analysis of the GATA transcription factor gene family in Ustilaginoidea virens. Genome. 2019;62(12):807-16. https://doi.org/10.1139/gen-2018-0190.

Zhang H, Liu K, Zhang X, Song W, Zhao Q, Dong Y, et al. A two-component histidine kinase, MoSLN1, is required for cell wall integrity and pathogenicity of the rice blast fungus, Magnaporthe oryzae. Curr Genet. 2010;56(6):517-28. https://doi.org/10.1007/s00294-010-0319-X.

Zhang H, Zhao Q, Guo X, Guo M, Qi Z, Tang W, et al. Pleiotropic function of the putative zinc-finger protein MoMsn2 in Magnaporthe oryzae. Mol Plant-Microbe Interact. 2014;27(5):446-60. https://doi.org/10.1094/MPMI09-13-0271-R.

Zheng DW, Wang Y, Han Y, Xu JR, Wang CF. UvHOG1 is important for hyphal growth and stress responses in the rice false smut fungus Ustilaginoidea virens. Sci Rep. 2016;6(1):24824. https://doi.org/10.1038/srep24824.

Zheng MT, Ding H, Huang L, Wang YH, Yu MN, Zheng R, et al. Low-affinity iron transport protein Uvt3277 is important for pathogenesis in the rice false smut fungus Ustilaginoidea virens. Curr Genet. 2017;63(1):131-44. https://doi. org/10.1007/s00294-016-0620-4.
Ready to submit your research? Choose BMC and benefit from:

- fast, convenient online submission

- thorough peer review by experienced researchers in your field

- rapid publication on acceptance

- support for research data, including large and complex data types

- gold Open Access which fosters wider collaboration and increased citations

- maximum visibility for your research: over $100 \mathrm{M}$ website views per year

At BMC, research is always in progress.

Learn more biomedcentral.com/submissions 